\title{
Programas preventivos promocionales de salud bucal en el Perú.
}

\author{
Promotional preventive oral health programs in Peru.
}

\author{
Erika Marisol Espinoza-Usaqui ${ }^{1, a}$, Flor de María Pachas-Barrionuevo ${ }^{1, b}$
}

\section{RESUMEN}

El artículo de revisión hace referencia a los programas preventivos promocionales de salud bucal, partiendo de la identificación de la problemática de salud bucal, con revisión de conceptos de los determinantes de la salud, prevención y promoción. Se explican los componentes de un programa en el que se incluyen objetivos, metas, técnicas y estrategias, actividades, recursos, etapas y además se analiza la propuesta de la OMS. La prevención con flúor vía sistémica y tópica y la aplicación de los sellantes de fosas y fisuras fueron las estrategias más utilizadas por los programas. Por su eficiencia y eficacia, la fluorización de sal de consumo humano y del agua potable, constituyen los programas con más impacto sobre la salud oral, según experiencias reportadas en diversos países de Europa, Asia, América y el Caribe. En el Perú, el programa empleado es la Sal Fluorada a cargo del Ministerio de Salud (MINSA). Los programas con fuerte contenido promocional basado en los principios de la promoción de la salud pretenden de un lado implementar políticas saludables en el cuidado de la salud, crear espacios saludables con énfasis en escuelas, orientar los servicios de salud a lo preventivo, organizar a la población y modificar conductas de riesgo a través de acciones de instrucción de higiene oral, disminución del consumo de carbohidratos, uso de pasta dental con flúor y visitas al dentista. Se obtuvo mejores resultados en la mejora de la salud bucal, cuando se emplearon ambos componentes, preventivo y promocional.

Palabras clave: SALUd BUCAL, PREVENCIÓn, PROMOCIÓN, PROGRAMAS DE SALUD.

\begin{abstract}
\footnotetext{
Facultad de Estomatología. Universidad Peruana Cayetano Heredia. Lima, Perú.
}

a Bachiller en Estomatología.

b Docente del Departamento Académico de Odontología Social.
\end{abstract}

This review article refers to Preventive Promotional Programs of Oral Health, starting from the identification of oral health problems, with a review of the concepts of health determinants, prevention and promotion. The components of a program are explained, including objectives, goals, techniques and strategies, activities, resources, stages and the WHO proposal is also analyzed. Prevention with systemic and topical fluoride, and the application of pit and fissures sealants were the strategies most used by the programs. By their efficiency and effectiveness, the fluoridation of salt for human consumption and drinking water, are the programs with the greatest impact on oral health, as experiences reported in various countries of Europe, Asia, America and the Caribbean. In Peru, the program used is Salt Fluorization in charge of The Ministry of Health (MINSA). Programs with strong promotional content based on the principles of health promotion, intend to 
implement healthy policies in health care, create healthy spaces with emphasis on schools, guide health services towards prevention, organize the population and modify risk behaviors through actions of oral hygiene instructions, reduction of sugar intake, use of fluoride toothpaste and dental visits. When both, preventive and promotional components were used, better results in oral health were obtained.

\section{Key words: ORAL HEALTH / PREVENTION / PROMOTION / HEALTH PROGRAMS}

\section{INTRODUCCIÓN}

Los programas de salud surgen para mejorar las condiciones de salud de aquellos problemas catalogados de impacto en salud pública. Están dirigidos generalmente a determinados grupos poblacionales y se miden por la eficacia o eficiencia, respecto al logro de sus objetivos planteados y empleo de los recursos al menor costo posible. En salud bucal, se organizan y ejecutan diversos programas, algunos con componentes promocionales y otros preventivos, $\mathrm{u}$ ambos desarrollados a la vez. Se miden por la variación de los índices de salud bucal e incremento de conocimientos o actitudes hacia la salud entre otros. Generalmente, el componente promocional está compuesto por acciones de fomento, educación para la salud y de empleo de estrategias de la promoción de la salud, mientras el componente preventivo emplea generalmente el uso de flúor por distintas vías o colocación de sellantes de fosas y fisuras. Los principales responsables de estos programas son los gobiernos y en algunos casos empresas o entidades privadas $\mathrm{u}$ organismos no gubernamentales quienes colaboran en esta noble intención.

\section{CONCEPTOS BÁSICOS}

La organización mundial de la salud OMS define a la salud bucodental como la ausencia de dolor orofacial crónico (1). En 1948, la Salud, fue definida como un estado de completo bienestar físico, mental y social y en 1986, surge otro concepto de salud como "recurso para la vida y no como objetivo de vida" $(2,3)$.

Los determinantes de la salud son un conjunto de factores personales, sociales, económicos y ambientales que determinan el estado de salud de los individuos o poblaciones. $(3,4)$. La salud púbica bucal se define como la ciencia y la práctica en la prevención de enfermedades bucales, promoción de la salud bucal y el mejoramiento de la calidad de vida, a través de los esfuerzos organizados de la sociedad $(5,6)$.
Un problema de salud pública se determina a través de cuatro criterios: Prevalencia de la condición, impacto de la condición a nivel individual, impacto en la sociedad y si la condición se puede prevenir y se dispone de tratamientos efectivos para ello (6).

Caplan, en 1980, clasifica los niveles de acción preventiva en: prevención primaria (evita la enfermedad), prevención secundaria (diagnóstico y tratamiento o curación) y prevención terciaria (rehabilitación). Asimismo, Gordon en 1987, contempla otros tipos de prevención: prevención universal (dirigida a la población), selectiva (dirigida a un segmento concreto de la población) e indicada (dirigida a personas que padecen los problemas objeto de la prevención). También, existen otros tipos de prevención en función del ámbito de intervención: la escuela, la familia, la comunidad, el trabajo, entre otros (6).

En 1986, la Carta de Ottawa, establece cinco campos centrales de acción: la elaboración de políticas públicas saludables, la creación de ambientes favorables para la salud, el empoderamiento comunitario, el desarrollo de habilidades personales y la reorientación del sistema de salud, que alude a una concepción global e intersectorial de salud $(2,3)$.

Los programas son definidos, como el diseño de un conjunto de actuaciones, entre sí relacionados, para la obtención de una meta. Para la OPS, "un programa se elabora para atender en forma continua un conjunto delimitado de problemas o necesidades de grupos específicos de la población estableciendo áreas de concentración con el fin de optimizar la utilización de recursos existentes para el logro de objetivos desagregados en ámbitos concretos" $(2,9,10)$.

Todo programa se compone de los siguientes elementos: propósitos, objetivos, metas, técnicas y/o estrategias, actividades, recursos, ámbitos, beneficiarios, fases o etapas, cronograma $\mathrm{y}$ responsables (Tabla 1) (2). 
Tabla 1. Componentes de un programa preventivo promocional de salud bucal.

\begin{tabular}{ll}
\hline COMPONENTES & \multicolumn{1}{c}{ DESCRIPCIÓN } \\
\hline Propósitos & $\begin{array}{l}\text { Aluden a los valores y a las intenciones } \\
\text { del programa. }\end{array}$ \\
Objetivos & Son declaraciones que describen las \\
& transformaciones que se pretenden lograr \\
& sobre situaciones existentes. \\
Metas & Son objetivos altamente especificados \\
& en magnitud y tiempo. Son perspectivas \\
& mensurables tanto cuantitativamente \\
& como cualitativamente, coherentes con \\
& los objetivos propuestos. \\
Técnicas y & Se refiere a cómo se llevarán a cabo los \\
Estrategias & objetivos del programa. Es decir, son las \\
& diversas maneras de proceder para poner \\
& en marcha el programa en las mejores \\
& condiciones posibles. \\
& $\begin{array}{l}\text { Son las diversas tareas mensurables, tan- } \\
\text { gibles y recurrentes que tienen en común }\end{array}$ \\
& conseguir un objetivo específico. Duran- \\
te el desarrollo de actividades, se hace el & monitoreo que es un proceso técnico ad- \\
& ministrativo que se realiza sobre activida- \\
& des claves, de forma continua. En cuanto \\
& a evaluación, se efectúan a dos niveles, a) \\
& Evaluación de resultados, que se da al fi- \\
& nal del programa. b) Evaluación del pro- \\
& ceso, que se realiza durante la ejecución \\
& del programa.
\end{tabular}

Recursos Se refiere a con qué se llevará a cabo la propuesta, el empleo de insumos tanto humanos como materiales, medios financieros e infraestructura.

Ámbitos Se refiere en dónde se implementará el programa.

Beneficiarios y/o Responde a quién fue pensado y orientaDestinatarios do el programa.

Fases o etapas Incluyen desde la evaluación inicial o diagnóstico, el desarrollo de las actividades y la evaluación final o resultados.

Cronograma Es un esquema donde se distribuye y organiza en forma de secuencia temporal el conjunto de actividades programadas.

Responsables

Alude a quienes responden y sostiene el programa.

\section{Programas Preventivos de Salud Bucal}

Los programas preventivos de salud bucal hacen énfasis en evitar la aparición de caries dental. La OMS, señala que se puede lograr una disminución en la incidencia de caries dental en las poblaciones que reciben flúor en niveles entre 0,7 y 1,49 ppm. Este elemento, flúor, se puede administrar a las personas de diferentes formas, ya sean éstas por vía tópica o sistémica, pero se ha demostrado que las más efectivas son las segundas y dentro de ellas la fluorización del agua y de la sal, por ser más masivas en su cobertura y más eficientes $(7,10,11)$.

Las medidas de prevención más usadas son las siguientes, y se organizan a través de programas:

Programa de fluorización del agua de consumo La OMS recomienda como valor guía para fluoruro en aguas el de $1,5 \mathrm{mg} / \mathrm{L}$. Se aplica a nivel de la comunidad, para lo cual es necesaria la adición del flúor en todos los acueductos del país. Sin embargo, no a todas las personas y regiones les llega el agua por acueducto, pues un gran porcentaje lo recibe de pozos individuales y otras fuentes, lo cual las excluiría de este programa de prevención. Además, no toda el agua que llega por los acueductos, y que estaría fluorada, es utilizada para el consumo, sino que una gran parte se destina a otros usos domésticos, industriales, agrícolas, Por estas razones se descarta el agua como posible vehículo del flúor en ciertos países $(3,7,11,12)$.

Desde 1945, numerosos países han practicado la fluorización del agua, siendo los países beneficiados: Estados Unidos, Canadá, Europa, ciertos países de Asia y América Latina como Chile, Argentina y Puerto Rico (11).

\section{Programa de fluorización de la sal de consumo}

La sal es adquirida y consumida por toda la población, lo cual garantiza una amplia cobertura de esta medida preventiva. Asimismo, es utilizada para el consumo de manera directa o indirecta, la sal constituye una medida preventiva muy eficiente. Es importante tener en cuenta que la dosis diaria de sal necesaria para las personas es de 0.05 a $0.07 \mathrm{mg} / \mathrm{kg}$ del peso corporal (11).

En Europa se han reconocido las ventajas de la fluorización de la sal en la ejecución eficaz de una política de prevención masiva de la caries dental durante 30 años. Hay pruebas científicas claras de una reducción estadísticamente significativa de la caries dental $(11,13,14)$.

En el Perú, el programa de fluorización de la sal de consumo se inició en 1985, por decreto supremo se exigió a las empresas dedicadas al procesamiento de la 
sal, añadir flúor a este producto. En 1988 el ministerio de salud MINSA recibe el apoyo de la Fundación KW Kellogg para la implementación de este programa y en 1993 se inicia el programa de fluorización de la sal de consumo humano en el Perú (15).

Programa de fluorización de la leche. Son programas preventivos comunitarios. Estos programas de fluorización en la leche han demostrado ser exitosos, habiendo sido aplicados en países como Suecia y Chile $(3,16)$.

Programa de fluorización mediante colutorios. Se utiliza una concentración de $0.02 \%$ de solución de fluoruro de sodio en enjuagues bucales semanales o quincenales. También se puede utilizar solución de fluoruro de sodio al 2\% combinado con laserterapia. Y solución o gel de fluoruro de sodio al $0.2 \%$ y clorhexidina al $0.2 \%$, utilizándose en enjuagatorios bucales o en el cepillado dental $(3,7,17)$.

En el Perú se implementó un programa de enjuagatorios con una solución fluorada al $0.2 \%$. en niños de 6 a 12 años, en centros educativos estatales de nivel primario y en direcciones de salud, hospitales, redes, micro redes y establecimientos de salud de la red de servicios del ministerio de salud (15).

Fluorización mediante el uso de pasta dentífrica Se emplean cremas dentales con fluoruro de sodio, monofluorfosfato o ambos, a concentraciones de 500 ppm para niños hasta los 10 años en territorios con flúor sistémico en el agua o sal, y en concentraciones mayores a este valor para el resto. Se utiliza con el cepillado diario $(3,7)$.

Aplicación de fluoruros por profesionales. Se utiliza gel de fluoruro de sodio neutro al $1.1 \%$ o al $2 \%$ y gel de fluorfosfato acidulado al $1.23 \%$. En una revisión hecha por Marinho y col, en el 2002-2004, los fluoruros tópico en gel logran una reducción de caries en un $28 \%$ y la aplicación de barnices un $46 \%$ $(3,8,18)$.

Aplicación de sellantes de fosas y fisuras. El uso de sellantes de fosas y fisuras se recomienda para la prevención de caries dental, ya que al cubrir con una capa plástica de seguridad las irregularidades del esmalte, mantiene sin efecto la acción bacteriana y la de los alimentos que causan la caries dental $(3,19,20,21)$.
Diversos autores han reportado resultados con disminución hasta de 72,8\% de dientes cariados, perdidos y obturados con programas preventivos sustentados en la aplicación de fluoruros, la colocación de sellantes y la fluorización de las aguas de consumo (Tabla 2).

\section{PROGRAMAS PROMOCIONALES DE SALUD BUCAL}

Se ha demostrado que los programas promocionales implementados por dentistas y profesores calificados incrementan significativamente la efectividad de las medidas para la prevención de la caries y gingivitis $(22,23,24,25)$ (Tabla 3).

En la práctica e implementación de programas, podemos encontrar programas de prevención con algunos componentes de promoción y viceversa (2).

\section{PROGRAMA DE SALUD BUCAL DE LA OMS/ OPS}

La (OMS), en colaboración con la comunidad internacional de salud bucal, coordina alianzas globales con el fin de compartir responsabilidades para implementar una estrategia global (1).

Las actividades de la OMS abarcan la promoción, la prevención y el tratamiento, como son:

- La promoción desde un enfoque basado en los factores de riesgo comunes.

- Los programas de fluorización para mejorar el acceso a los fluoruros en los países de bajos ingresos.

- El apoyo técnico a los países que están integrando la salud bucodental en sus sistemas de salud pública (7). A través del marco de escuelas de promoción de la salud de la OMS, desde 1995, diseña programas para mejorar la salud de niños, personal escolar, familias y otros miembros de la comunidad a través de los colegios. Esta iniciativa consiste en cuatro amplias estrategias:

- Capacidad de interceder en programas de salud escolar mejorados.

- Crear redes y alianzas para el desarrollo de escuelas promotoras de la Salud.

- Fortalecimiento de la capacidad nacional.

- Investigar para mejorar los programas de salud en las escuelas $(25,26)$. 
Tabla 2. Resultado de programas preventivos de salud bucal.

\begin{tabular}{|c|c|c|}
\hline PROGRAMA & Autor / País & Resultados \\
\hline Fluorización del Agua & Locker 1999 y Mc Donagh 2000 & $\begin{array}{l}\text { Encontraron una reducción de caries en el 14\% y un mejor } \\
\text { efecto en la dentición primaria. }\end{array}$ \\
\hline Fluorización de Sal & $\begin{array}{l}\text { Jamaica } \\
\text { Costa Rica } \\
\text { Cuba }\end{array}$ & $\begin{array}{l}\text { Se logró una reducción de la caries dental en una } 85 \% \text { ( } 8 \text { años) } \\
\text { Se logró una reducción de } 40 \% \text { ( } 5 \text { años de ejecutado). } \\
\text { Se logró una reducción de } 40 \% \text { ( } 5 \text { años) y } 80 \% \text { (luego de } 10 \\
\text { años), en menores de } 15 \text { años de edad. } \\
\text { Se logró una disminución hasta } 40 \% \text { en los de } 15 \text { y más años } \\
\text { de edad. Se logró un incremento de los pacientes sanos en } \\
\text { más del } 20 \% \text { a los } 5 \text { años de iniciado el programa. Se logró el } \\
\text { acceso de la población a los beneficios del programa, superior } \\
\text { al } 95 \% \text {. } \\
\text { Se logró una disminución en más del } 25 \% \text { de los costos en la } \\
\text { atención curativa a los menores de } 15 \text { años de edad, a los } 5 \text { años } \\
\text { del programa. }\end{array}$ \\
\hline F. Leche & Espelid 2009 & $\begin{array}{l}\text { Encontró una reducción de } 78 \% \text { de caries en dientes permanen- } \\
\text { tes recién erupcionados en niños de } 8 \text { años los cuales habían } \\
\text { consumido leche fluorada por } 3 \text { años y también hubo una reduc- } \\
\text { ción de } 31 \% \text { para dientes primarios. }\end{array}$ \\
\hline F. Colutorio & $\begin{array}{l}\text { Borroto y Col. } 2003 \\
\text { Marinho 2002-2004 }\end{array}$ & $\begin{array}{l}\text { Encontraron una reducción de } 75 \% \text { en premolares y dientes an- } \\
\text { teriores, } 43 \% \text { en molares, } 88 \% \text { en superficies proximales, } 47 \% \\
\text { en las superficies oclusales. } \\
\text { Encontró una reducción en un } 26 \% \text { de la caries dental. }\end{array}$ \\
\hline F.Pasta Dental & Marinho 2002-2004 & Encontró una reducción en un $24 \%$ de la caries dental. \\
\hline F. Gel & Marinho y col 2002-2004 & Encontraron una reducción en un $28 \%$ de la caries dental. \\
\hline Sellantes & $\begin{array}{l}\text { Ahovou-saloranto } 2004 \\
\text { Parnell y Col. } 2003\end{array}$ & $\begin{array}{l}\text { Encontró una reducción en un } 86 \% \text { y } 57 \% \text { de la caries dental, a } \\
\text { los } 12 \text { y } 48 \text { meses respectivamente. } \\
\text { Encontraron, luego de } 3 \text { años, que el } 56 \% \text { de sellantes perma- } \\
\text { necían completos en boca y que la experiencia de caries en los } \\
\text { dientes sellados fue de } 2.9 \% \text {. }\end{array}$ \\
\hline $\begin{array}{l}\text { Aplicación de flúor, sellan- } \\
\text { tes, Flúor en el agua }\end{array}$ & $\begin{array}{l}\text { Dini y Silva } 1994 \\
\text { Horowitz } 1984\end{array}$ & $\begin{array}{l}\text { Reportaron resultados con disminución hasta de } 72,8 \% \text { de dien- } \\
\text { tes cariados, perdidos y obturados. }\end{array}$ \\
\hline
\end{tabular}

Asimismo, se han desarrollado programas para gestantes, cuyo fundamental objetivo ha sido el de contribuir a elevar el nivel de salud bucal de las gestantes con acciones de promoción, protección y recuperación de la salud de la mujer embarazada $(25,27)$.

La Organización Panamericana de la Salud (OPS) desarrolló el Plan Regional Decenal sobre Salud Bucodental. En él se definen las estrategias y metas para los países de la región, con base al índice de dientes cariados, perdidos y obturados a los 12 años (28).

La OPS tiene un plan de varios años para los programas de fluorización del agua y de la Sal; y ha propuesto tres etapas en la ejecución: evaluación de la factibilidad, evaluación a corto plazo y evaluación a largo plazo (28).

La metas propuestas por la OPS son: Iniciar evaluaciones de la factibilidad (costo-beneficio) y del punto de comparación para los programas nacionales de fluorización del agua y de la sal en seis países; apoyar el fortalecimiento de la capacidad de los países para implantar sistemas eficaces de vigilancia epidemiológica; apoyar la elaboración de material didáctico sobre salud bucal adaptado al programa de estudios; continuación de los estudios de factibilidad y las evaluaciones para otros programas nacionales; apoyar el establecimiento de programas sostenibles de fluorización en todos los países; y reforzar la educación en salud oral y los materiales del programa $(28,29)$.

\section{PROGRAMAS PREVENTIVO PROMOCIONALES EN EL PERÚ}

El Ministerio de Salud ha desarrollado ciertas estrategias para la prevención de la caries dental. Dentro de las cuales tenemos a la fluorización de la 
Tabla 3. Resultados de Programas Promocionales de Salud Bucal.

\begin{tabular}{|c|c|c|}
\hline PROGRAMA & Autor / País & Resultados \\
\hline $\begin{array}{l}\text { Programa Piloto } \\
\text { Campaña Televisiva }\end{array}$ & Friel y Col. 2002 (Irlanda) & $\begin{array}{l}\text { Después de } 6 \text { semanas, se encontró un incremento en } \\
\text { el porcentaje de niños que reportaron usar la canti- } \\
\text { dad recomendada de pasta dental y cepillarse por } 3 \\
\text { minutos. }\end{array}$ \\
\hline Programa de Promoción de salud bucal & Australia 2007 & $\begin{array}{l}\text { Reducción en el sufrimiento innecesario como hospita- } \\
\text { lización y tratamientos invasivos en niños de } 2 \text { a } 3 \\
\text { años. }\end{array}$ \\
\hline $\begin{array}{l}\text { Programa de promoción de salud bucal } \\
\text { para padres de infantes }\end{array}$ & Plutzer K, Soencer AJ 2007 & $\begin{array}{l}\text { El resultado fue una incidencia de S-ECC de } 1.7 \% \text { en } \\
\text { las madres que recibieron el programa y de } 9.6 \% \text { en } \\
\text { las que no la recibieron. }\end{array}$ \\
\hline $\begin{array}{l}\text { Programa preventivo educacional en la } \\
\text { salud bucal de niños preescolares }\end{array}$ & Hochstetter y Col. en el 2007 & $\begin{array}{l}\text { En los resultados a los } 8 \text { días y después de } 6 \text { y } 12 \text { me- } \\
\text { ses, se obtuvo una disminución significativa en el ín- } \\
\text { dice gingival e índice de placa, y no hubo incremento } \\
\text { significativo en caries dental en el grupo experimental. }\end{array}$ \\
\hline
\end{tabular}

sal de consumo humano, iniciándose en el año 1984 por Decreto Supremo $(30,31)$.

Asimismo, en el 2001, se aprueba la Norma técnica Sanitaria para la adición de fluoruros en cremas dentales, enjuagatorios y otros productos utilizados en la higiene bucal, la cual pretende establecer especificaciones técnicas que garanticen la calidad del producto y regule las concentraciones adecuadas de flúor con el objetivo de prevenir las caries y la fluorosis dental (32).

En el 2001, la Dirección General de Salud de las Personas mediante la directiva 005-2001, reguló la aplicación de Ionómero de vidrio mediante la Técnica de Restauración Atraumática (TRA), con el fin de ofrecer una alternativa de restauración a piezas dentarias cariadas mediante el uso de instrumentos manuales; sin embargo, dicha técnica encontró dificultades en el proceso de retiro de dentina cariada, como en la colocación del ionómero de vidrio autocurable (33).

El plan nacional concertado de salud 2007-2020 menciona como objetivo sanitario disminuir las enfermedades de la cavidad bucal, reducir el índice de CPOD y la inclusión de gestantes en la atención preventiva dental. La comisión del programa nacional de salud bucal, mediante un conjunto de actividades refuerza e implementa hábitos de higiene oral, el uso correcto de flúor y fomenta el consumo de sal fluorada en la población, y en especial en las poblaciones de riesgo como los pre-escolares, escolares y gestantes (31). El Plan Nacional de salud bucal en escenarios educativos y el Plan Nacional de Salud Madre Niño están dirigidos a mejorar la calidad de la salud bucal de la madre gestante y el bebé (30).

La ejecución del programa promueve el auto cuidado con buenas prácticas de higiene bucal en la población escolar, preferentemente las más pobres del país; se implementan metodologías de comunicación educativa ejecutadas por las Comisiones de Salud Bucal, Círculos Odontológicos Distritales y Provinciales de las diferentes regiones del país para lo cual se proponen diferentes herramientas metodológicas interpersonales y masivas (30).

En el Perú, EsSalud, Sanidad de las Fuerzas Armadas, Fuerzas Policiales, Empresas o entidades privadas $\mathrm{u}$ organismos no gubernamentales vienen brindando los cuidados a estas necesidades a través de la atención individual (consejería) y/o colectiva (charlas educativas), estos cuidados están siendo incorporados recientemente en los paquetes de atención integral del sector $(30,35,36,37,38)$.

\section{CONCLUSIÓN}

Los programas preventivos que usan flúor por vía sistémica son eficaces y tienen un alto costobeneficio. Sin embargo, el empleo de programas de fluorización con sal tiene mayor posibilidad de cubrir a toda la población.

Los programas promocionales demuestran eficacia en el mejoramiento de conocimientos y actitudes al estar dirigidos a niños, padres de familia y profesores.

Los programas que combinan ambos componentes 
preventivos y promocionales han logrado resultados más eficaces, que aplicados individualmente. Por ello, es importante que todo programa tenga ambos componentes.

Asimismo, todas las instituciones y profesionales de la salud en el Perú, deben tener en consideración estos aspectos para la implementación de nuevos programas de salud bucal y la mejora continua de los mismos, estableciéndose programas preventivo promocionales bien elaborados, y a su vez un sistema de vigilancia y de monitoreo que pueda evaluar la evolución de estos programas a lo largo de los años. La reducción de ellas no solo implica un beneficio individual para la persona, sino que involucra toda una cadena de beneficios para el país, al reducir los costos en tratamientos y por ende el presupuesto que el gobierno asigna para la salud. Ya que la salud es fundamental para el bienestar humano y el desarrollo económico y social sostenible, por lo tanto la salud bucal es un derecho y debe ser apoyado por los gobiernos.

\section{CORRESPONDENCIA}

Erika M. Espinoza Usaqui

Calle Schell 275 Dpt.304-Lima 18. Perú.

Teléfono: 993407837

e-mail: erikaespinozau@hotmail.com

\section{REFERENCIAS BIBLIOGRÁFICAS}

1. OMS. Salud Bucodental. Centro de prensa. Nota informativa $\mathrm{N}^{\mathrm{a}}$ 318.Febrero 2007[acceso12 de Enero del 2012]. Disponible en: http://www.who.int/mediacentre/ factsheets/fs318/es/

2. Vincezi A, Tudesco F. La educación como proceso de mejoramiento de la calidad de vida de los individuos y de la comunidad. Revi Iberoam Educ [revista online] 2009[acceso 12 de Enero del 2012];49(7):1-12.Disponible en: www.rieoei. org/ deloslectores/2819Vicenzi.pdf

3. Watt RG. Strategies and approaches in oral disease prevention and health promotion. Bull World Health Organ [revista online] 2005 [acceso12 de Enero del 2012];83(9):711-8. Disponible en : http://www.who.int/bulletin/volumes/83/9/711. pdf

4. Sheiham A. Improving oral health for all: focusing on determinants and conditions. Health Educ J. 2000;59(4):35163

5. Universidad de Sydney. Glosario OMS. Promoción de la Salud Glosario. [acceso12 de Enero del 2012];Australia.1998. 1-35 pag. Disponible en: http://www.who.ch/hep

6. Daly B, Watt RG, Batchelor P, Treasure ET. Essential Dental Public Health: Oxford University Press; 2003.

7. OMS. The objectives of the WHO Global Oral Health
Programme (ORH). [acceso 12 de Enero del 2012 ]. Disponible en :http:/www.who.int/oral health/ objectives/ en/index.html

8. Petersen PE. Challenges to improvement of oral health in the 21st century-the approach of the WHO Global Oral Health Programme. Int Dent J. 2004;54:329-43.

9. Community Liaison Division Department of health. Basic principles of healthy cities health for all .2009 [acceso 22de Enero del 2012] Disponible en:http://www.chp.gov.hk/files/ pdf/basic_principles_of_healthy_cities-health_for_all.pdf

10.Hobdell M, Petersen PE, Clarkson J, Johnson N. Global goals for oral health 2020. Int Dent J. 2003;53:285-88 [acceso 22de Enero del 2012] Disponible en: http://www.who.int/oral health/media/en/orh_goals_2020.pdf

11. Sosa M. Evolución de la fluorización como medida para prevenir la caries dental. Rev Cubana Salud Pública. 2003;29(3):268-74. Disponible en : http://bvs.sld.cu/revistas/ spu/vol29_3_03/spu11303.htm

12.Oral health: action plan for promotion and integrated disease prevention. Sixteth WorldHealth Assemby [revista en internet] 2007 [acceso19 de Febrero del 2012] Disponible en: http:// www.bfsweb.org/documents/A60_R17-en1.pdf

13.OPS. Salud Oral en Regiones. 2006 [acceso19 de Febrero del 2012] Disponible en: http://www.paho.org/Spanish/gov/cd/ doc259.pdf

14.Sosa M, García M, Gómez A, González I, Mojáiber A. Estrategia para la ejecución del Programa de Fluoruración de la Sal de Consumo en Cuba. Rev Cubana Salud Pública. 2004;30(3).

15.Ascue S. Ministro de Salud destaca Plan Nacional de Salud Madre-Niño. Actual Odontol Salud. 2010;7(1):3-4.

16.Stecksén-Blicks C, Sjöström I, Twetman S. Effect of longterm consumption of milk supplemented with probiotic lactobacilli and fluoride on dental caries and general health in preschool children: a cluster-randomized study. Caries Res. 2009;43(5):374-81. Epub 2009 Aug 18.

17. Borroto R y Saez M. Programa preventivo de caries dental en niños y adolescentes cubanos. Rev Cubana Estomatol [Revista en internet] 2003 [acceso 22 de Febrero del 2012];40(2). Disponible en: http://redalyc.uaemex.mx/pdf/ 912/ 91223410. pdf

18.Daley C, Gubb J. Healthcare Systems: Switzerland .CIVITAS Institute for the Study of Civil Society 2011.12/2007pag 1-14[acceso19 de Febrero del 2012] Disponible en: www. civitas.org.uk/nhs/download/switzerland.pdf

19.Parnell CA, O'Farrell M, Howell F, Hegarty M. Evaluation of a community fissure sealant programme in County Meath, Ireland. Community Dent Health. 2003 Sep;20(3):146-52.

20.Carrero G, Fleitas AT, Arrellano L. Prevención de caries dental en primeros molares permanentes utilizando sellantes de fosas y fisuras y enjuagues bucales fluorurados. Revista Odontológica de los Andes [revista en internet] 2006 [acceso 22 de Febrero del 2012];1. Disponible en: http://www.saber. ula.ve/bitstream/123456789/24807/2/ articulo5.pd

21.Memarpour M, Shafiei F, Shokouth P, Shaddel M. Evaluation of a school-based pit and fissure sealant programme in Iranian children. Oral Health Prev Dent. 2011;9(4):381-6.

22.Friel S, Hope A, Kelleher C, Comer S, Sadlier D. Impact evaluation of an oral health intervention amongst primary school children in Ireland. Health Promot Int 2002;17(2):119126.

23.Plutzer K, Spencer AJ. Efficacy of an oral health promotion 
intervention in the prevention of early childhood caries. Community Dent Oral Epidemiol. 2008;36:335-46.

24.Hochstetter AS, Lombardo MJ, Deramo L, Piovano S, Bordoni N. Effectiveness of a preventive educational programme on the oral health of preschool children. Promotion \& Education. 2007; $14: 155-8$.

25.WHO. Important tarjet groups. [accesol1 de Enero del 2012] Disponible en: http://www.who.int/oral_health/action/groups/ en/index 1.html

26. Moyses ST, Moyses SJ, Watt R, Sheiham A. Associations between health promoting schools ${ }^{\wedge} \mathrm{s}$ policies and indicators of oral health in Brazil. Health Promot Int. 2003;18(3):209-18.

27.Petrovic PM. Evaluación del Programa de Educación para la Salud en el tratamiento estomatológico de mujeres embarazadas en la ciudad de Nis-Serbia. Revista ADM. 2007;5:197-200.

28.PAHO. Oral Health in the Americas. Chapter 2: Health
Conditions and Trends [revista en internet] 2007 [acceso 19 de Febrero del 2012];135-141). Disponible en: http://www.paho. org/hia/archivosvol1/volregionaling/HIA07\%20Regional $\% 20$ Volume\%20ENG\%20Ch\%202.pdf

29.Salud Oral en Regiones. 2006 [acceso 19 de Febrero del 2012] Disponible en: http://www.paho.org/Spanish/gov/cd/doc259. pdf

30.Ministerio de Salud. Programa Nacional de Salud Bucal 2009.2010.Colegio Odontológico del Perú .[acceso 12 de Enero del 2012] Disponible en: http://es.scribd.com/ doc/15443456/Programa-Nacional-de-Salud-Bucal-Minsa

31.Cam LE. Fluorización de la sal en el Perú. 2006 [acceso 26 de Febreo del 2012] Disponible en: http://odontorural.wordpress. com/2006/08/24/fluorizacion-de-la-sal-en-el-peru/al

32. Ministerio de Salud. Norma Técnica Sanitaria de Calidad para las cremas dentales cosméticas. 2005 [acceso 26 de Febrero del 2012]Pag 3-8.Disponible en: http://www.google.

Recibido : 15-05-2012

Aceptado: 22-08-2012 